\title{
Unemployment Period Approach with Infinite Servers Queue Systems
}

\author{
Manuel Alberto M. Ferreira \\ Instituto Universitário de Lisboa (ISCTE-IUL), Lisboa, Portugal
}

\begin{abstract}
With results on the infinite servers queue systems with Poisson arrivals - $M|G| \infty$ queues - busy period, it is displayed an application of those queue systems in the unemployment periods time length parameters and distribution function study. These queue systems are adequate to the study of many population processes, and this quality is brought in here. The results presented are mainly on unemployment periods length and their number in a certain time interval. Also, some questions regarding the practical applications of the outlined formulas are briefly discussed.
\end{abstract}

Keywords: infinite servers queues, busy period, unemployment

\section{The Model Rising}

In the queue systems used thoroughgoing in this article, see for instance, Ferreira, Filipe, and Coelho (2016), the customers arrive, according to a Poisson process at rate $\lambda$ and receive a service which time length is a positive random variable with distribution function $G($.$) and mean \alpha$. When a customer arrives it finds immediately an available server ${ }^{1}$, each customer service is independent from the other customers' services and from the arrivals process and the traffic intensity is $\rho=\lambda \alpha$. That is: they are $M|G| \infty$ queues. It is easy to understand how these queues can be applied to the unemployment period study. Then $\lambda$ is the rate at which occur the firings, supposed to happen according to a Poisson process and the service time, paradoxically, is the time between the worker firing and his/her discovery of a new job.

In any queue system, a busy period is a period that begins when a costumer arrives at the system finding it empty, ends when a costumer abandons the system letting it empty and there is always at least one customer present. So in a queuing system, there is a sequence of idle and busy periods, during its operation time. In the $M|G| \infty$ queue system, as in any queue system with Poisson arrivals, the idle periods have an exponentially distributed length with mean $\lambda^{-1}$. But the busy period's distribution is much more complicated, being in general given by infinite sums which parcels are convolutions (Ferreira \& Andrade, 2009). Despite it, it is possible to present some results as it will be seen. For what interests in this work: a busy period is a period of unemployment and an idle period is a period of full employment.

The results to be presented are on unemployment periods length and their number in a certain time interval. A study in following also this approach, over a public health situation, can be seen in Ferreira (2014).

\footnotetext{
Manuel Alberto M. Ferreira, Full Professor, ISTAR-IUL, BRU-IUL, Instituto Universitário de Lisboa (ISCTE-IUL). Email: manuel.ferreira@iscte.pt.

${ }^{1}$ Or there is no distinction between the customer and its server, as it happens in the application to be considered in this work.
} 


\section{Unemployment Period Time Length Distribution}

Designate $D$ the random variable unemployment period length. According to the results known for the $\mathrm{M}|\mathrm{G}| \propto$ queue busy period length distribution, see Ferreira and Andrade (2009):

$$
E[D]=\frac{e^{\rho}-1}{\lambda}
$$

whichever is the worker unemployment time length distribution, see Takács (1962).

As for $\operatorname{Var}[D]$, it depends on the whole unemployment time length distribution probabilistic structure. But Sathe (1985) demonstrated that:

$$
\max \left[e^{2 \rho}+e^{\rho} \rho^{2} \gamma_{s}^{2}-2 \rho e^{\rho}-1 ; 0\right] \leq \lambda^{2} \operatorname{Var}[D] \leq\left[2 e^{\rho}\left(\gamma_{s}^{2}+1\right)\left(e^{\rho}-1-\rho\right)-\left(e^{\rho}-1\right)^{2}\right]
$$

where $\gamma_{s}$ is the unemployment time length coefficient of variation.

For $\alpha$ and $\rho$ great enough (very intense unemployment conditions) since $G($.$) is such that for \alpha$ great enough $G(t) \cong 0, t \geq 0$ :

see Ramalhoto and Ferreira (1996).

$$
D(t) \cong 1-e^{-\lambda e^{-\rho} t}, t \geq 0
$$

Calling $N_{D}$ the mean number of unemployed people in the unemployment period, if $G($.$) is an exponential$ distribution:

$$
N_{D}=e^{\rho}
$$

For any other $G($.$) , probability distribution:$

$$
N_{D} \cong \frac{e^{\rho\left(\gamma_{s}^{2}+1\right)}\left(\rho\left(\gamma_{s}^{2}+1\right)+1\right)+\rho\left(\gamma_{s}^{2}+1\right)-1}{2 \rho\left(\gamma_{s}^{2}+1\right)}
$$

see Ferreira and Filipe (2010). Of course, multiplying Equation (2.4) or (2.5), as appropriate, by the mean cost of each unemployment subsidy, it is possible to estimate the assistance costs caused by the unemployment period.

\section{Unemployment Periods in a Time Interval Mean Number}

After the renewal processes theory (see Çinlar, 1975), calling $R(t)$ the mean number of unemployment periods that begin in $[0, t]$, being $t=0$ the beginning instant of an unemployment period, it is possible to obtain (see Ferreira, 1995):

$$
R(t)=e^{-\lambda \int_{0}^{t}[1-G(v)] d v}+\lambda \int_{0}^{t} e^{-\lambda \int_{0}^{u}[1-G(v)] d v} d u
$$

and consequently, the following simple upper and lower bounds for $R(t)$ (see Ferreira, 2004):

$$
e^{-\rho}(1+\lambda t) \leq R(t) \leq 1+\lambda t
$$

\section{Conclusions}

So that this model can be applied, it is necessary that the firings occur according to a Poisson process at a constant rate. It is a hypothesis that must be tested. Thus remain outside of this study, periods of unemployment caused by mass firings. 
Among the results presented, Equations (2.1), (2.2), (2.3), (2.5), and (3.2) are remarkable for its simplicity and for requiring only the knowledge of the firings rate $\lambda$, the mean unemployment time $\alpha$, and the unemployment time variance.

\section{References}

Çinlar, E. (1975). Introduction to stochastic processes. Englewood Cliffs, NJ: Prentice Hall.

Ferreira, M. A. M. (1995). Comportamento transeunte e período de ocupação de sistemas de filas de espera sem espera (Ph.D. thesis, ISCTE-IUL, Lisboa).

Ferreira, M. A. M. (2004). M $|\mathrm{G}| \propto$ queue busy cycle renewal function for some particular service time distributions. Proceedings of the International Conference Quantitative Methods in Economics (Multiple Criteria Decision Making XII) (M. Lukáèik, ed.), Virt, Slovakia, 2004, pp. 42-47.

Ferreira, M. A. M. (2014). The pandemic period length modelled through queue systems. Proceedings of the International Conference Quantitative Methods in Economics (Multiple Criteria Decision Making XVII) (R. Marian, P. Gezik, \& L. Martin, eds.), Virt, Slovakia, 2014, pp. 43-47.

Ferreira, M. A. M., \& Andrade, M. (2009). The ties between the M/G/oo queue system transient behaviour and the busy period. International Journal of Academic Research, 1(1), 84-92.

Ferreira, M. A. M., \& Filipe, J. A. (2010). Economic crisis: Using $\mathrm{M}|\mathrm{G}| \infty$ queue systems busy period to solve logistics problems in an organization. China-USA Business Review, 9(9), 59-63.

Ferreira, M. A. M., Filipe, J. A., \& Coelho, M. (2016). Unemployment period quantitative approach through infinite servers queue systems. Proceedings of the International Conference Quantitative Methods in Economics (Multiple Criteria Decision Making XVIII) (R. Marian \& P. Gezik, eds.), Vratna, Slovakia, 2016, pp. 89-94.

Ramalhoto, M. F., \& Ferreira, M. A. M. (1996). Some further properties of the busy period of an $\mathrm{M}|\mathrm{G}| \infty$ queue. Central European Journal for Operations Research and Economics, 4(4), 251-278.

Sathe, Y. S. (1985). Improved bounds for the variance of the busy period of the $\mathrm{M}|\mathrm{G}| \infty$ queue. Advances in Applied Probability, 17(4), 913-914.

Takács, L. (1962). An introduction to queueing theory. Oxford University Press. 